Article

\title{
Verification of Underlying Genetic Cause in a Cohort of Russian Patients with Familial Hypercholesterolemia Using Targeted Next Generation Sequencing
}

\author{
Anna E. Semenova ${ }^{1,2, *}$, Igor V. Sergienko ${ }^{1}$, Diego García-Giustiniani ${ }^{2}$, Lorenzo Monserrat ${ }^{2}$, \\ Anna B. Popova ${ }^{1}$, Diana N. Nozadze ${ }^{1}$ and Marat V. Ezhov ${ }^{1}$ \\ 1 National Medical Research Center of Cardiology, 121552 Moscow, Russia; igorcardio@mail.ru (I.V.S.); \\ anna.b.popova@gmail.com (A.B.P.); dana7.81@mail.ru (D.N.N.); marat_ezhov@mail.ru (M.V.E.) \\ 2 Health in Code SL, Clinical Department, 15006 A Coruña, Spain; diego.garcia@healthincode.com (D.G.-G.); \\ lorenzo.monserrat@healthincode.com (L.M.) \\ * Correspondence: an.sem@mail.ru; Tel.: +7-926-239-4171
}

Received: 27 February 2020; Accepted: 8 May 2020; Published: 14 May 2020

\begin{abstract}
Russian patients with familial hypercholesterolemia (FH) were screened for pathogenic mutations using targeted next generation sequencing. Genetic testing was performed in 52 probands with definite or probable FH based on the Dutch lipid clinic network criteria (DLCN score $\geq 6$ ). Blood samples were studied by massive parallel sequencing (Illumina HiSeq 1500 platform) using a custom capture library related to dyslipidemia and premature atherosclerosis. Mutations considered to be responsible for monogenic $\mathrm{FH}$ were identified in $48 \%$ of the probands: 24 with mutations in the $L D L R$ gene and two with a mutation in the $A P O B$ gene. There were 22 pathogenic/likely pathogenic mutations in $L D L R$, eight of which have not been previously described in the literature. Four patients with a clinical picture of homozygous FH had two heterozygous LDLR mutations. Although mutation-negative patients had highly elevated total cholesterol and low-density lipoprotein cholesterol levels, only half of them had a family history of hypercholesterolemia. With respect to heterozygous $\mathrm{FH}$, mutation-positive patients had higher maximum total cholesterol levels $(p=0.01)$, more severe carotid atherosclerotic lesions, and a higher percentage of premature peripheral artery disease $(p=0.03)$ than mutation-negative ones. However, the number of patients who suffered from myocardial infarction was similar between the two groups.
\end{abstract}

Keywords: familial hypercholesterolemia; next generation sequencing; LDLR; Dutch lipid clinic network criteria; cardiovascular risk

\section{Introduction}

Familial hypercholesterolemia $(\mathrm{FH})$ is a condition mostly associated with pathogenic mutations in the low-density lipoprotein receptor $(L D L R)$, apolipoprotein $\mathrm{B}(A P O B)$ and proprotein convertase subtilisin/kexin type 9 (PCSK9) genes. This disease is highly underdiagnosed and undertreated in the Russian Federation and its true prevalence remains unknown [1]. An analysis of the population in West Siberian, Russia, showed an estimated prevalence of about 1:400 for clinically diagnosed definite FH and 1:108 for both definite and probable FH in combination [2], which is consistent with the estimated prevalence of FH in Europe and the USA [3-6]. The recent development of Russian FH Registry is intended to bring more attention to the problem FH represents in Russia (ClinicalTrials.gov Identifier: NCT02208869) [7]. As of now, most of the genetic studies in Russian patients with FH have been performed as part of research programs before the era of next generation sequencing 
(NGS). Hence, the amount of publications is extremely limited. At the same time, a proper genetic study is essential to confirm FH. The evaluation of additional genes associated with dyslipidemia and premature atherosclerosis may help to identify additional genetic factors which could modify the phenotype [8].

The aim of this study was to investigate the presence of pathogenic mutations in a cohort of patients with definite or probable diagnosis of FH by using targeted NGS.

\section{Materials and Methods}

\subsection{Subjects}

The study included 52 unrelated patients with definite or probable FH based on the Dutch lipid clinic network criteria (DLCN score $\geq 6$ ) [3]. It was a cohort of patients originating from different regions of Russia who were currently living in Moscow and previously underwent a medical examination at the National Medical Research Center of Cardiology (NMRCC), Moscow, Russia. Signed informed consent was necessary to participate in the study. Men and women aged 18-75 years with total cholesterol (TC) $\geq 7.5 \mathrm{mmol} / \mathrm{L}$ or low-density lipoprotein cholesterol (LDL-C) $\geq 4.9 \mathrm{mmol} / \mathrm{L}$ were included. The exclusion criteria were hypothyroidism (thyroid-stimulating hormone $\geq 1.5 \mathrm{UNL}$ ), glycosylated hemoglobin $>9 \%$ and creatinine clearance $<30 \mathrm{~mL} / \mathrm{min}$. The lipid parameters measurements and carotid duplex ultrasound was performed as part of medical examination during the period of one year at the moment of selection for genetic study. The clinical information, current laboratory data and carotid arteries duplex ultrasound description were taken from clinical examination and medical records of laboratory and instrumental evaluation performed at NMRCC, Moscow, Russia. The study was approved by the ethics committee of the NMRCC.

\subsection{Genetic Study}

The blood samples were studied by massive parallel sequencing using a custom capture library. Coding exons and exon-intron boundaries of 63 genes (Table 1, Table S1) related to dyslipidemia and premature atherosclerosis were captured using a custom probe library (SureSelect Target Enrichment Kit for Illumina paired-end multiplexed sequencing method; Agilent Technologies, Santa Clara, CA, USA).

Table 1. List of 63 evaluated genes related to dyslipidemia and premature atherosclerosis.

\begin{tabular}{ll}
\hline \multicolumn{1}{c}{ Associated Phenotypes } & \multicolumn{1}{c}{ Genes } \\
\hline & ABCA1, ABCG1, ABCG5, ABCG8, ANGPTL3, \\
Monogenic dyslipidemias & APOA1, APOA5, APOB, APOC2, APOC3, APOE, \\
& CETP, GPD1, GPIHBP1, LCAT, LDLR, LDLRAP1, \\
& LIPA, LIPC, LMF1, LPA, LPL, LRP6, MEF2A, MTTP, \\
& MYLIP, PCSK9, PLTP, SAR1B, SCARB1, SLC25A40 \\
\hline \multirow{3}{*}{$\begin{array}{l}\text { Other inherited conditions related to } \\
\text { dyslipidemia and premature atherosclerosis }\end{array}$} & AGPAT2, AKT2, AMPD1, BLK, BSCL2, CAV1, CEL, \\
& CIDEC, COQ2, CPT2, CYP2D6, GCK, HNF1A, \\
& HNF1B, HNF4A, INS, INSR, KLF11, LEP, LMNA, \\
& PPAROD1, NPC1L1, PAX4, PDX1, PLIN1, PNPLA2, \\
& PPARG, PTRF, PYGM, SLC22A8, ZMPSTE24 \\
\hline
\end{tabular}

Sequencing was performed using the Illumina HiSeq 1500 platform (Illumina, San Diego, CA, USA) with $2 \times 100$ base pair read length following Illumina protocols. Bioinformatics analysis was performed by means of a custom pipeline that includes software as NovoAlign (Novocraft Technologies Sdn Bhd), SAMtools and BCFtools (Sanger Institute) for variant calling and genotyping and Annovar for variant annotation. This genetic test is aimed at identifying single nucleotide variants (SNVs) and small insertions/deletions up to $20 \mathrm{bp}$. Sanger sequencing was used to evaluate regions of low coverage and to confirm the genetic variants considered clinically relevant according to the patient's 
phenotype. The analytical sensitivity and accuracy is greater than $99 \%$. Copy number variations were also evaluated using a comparative depth-of-coverage strategy.

To establish the pathogenicity of the variants, we adopted a customized classification scheme based on the recommendations of the American College of Medical Genetics and Genomics (ACMG) (Table S2) [9]; information regarding frequency in different populations (1000 Genomes Project, Exome Variant Server, Exome Aggregation Consortium, Genome Aggregation Database [v2.1.1 version]) was considered. The final classification of each variant was agreed by consensus between two clinical cardiologists and genetics experts.

\subsection{Laboratory Analysis}

The TC, triglycerides (TG) and high-density lipoprotein cholesterol (HDL-C) serum levels were measured after 12-h fasting in NMRCC's certified laboratory at the time of inclusion in the study. LDL-C was calculated using the Friedewald formula [10]. TG levels were $<4.5 \mathrm{mmol} / \mathrm{L}$ in all patients. $\mathrm{Lp}$ (a) concentration was determined by enzyme-linked immunosorbent assay using monospecific polyclonal sheep anti-human-apo(a) antibodies as previously reported [11].

\subsection{Duplex Scanning of Carotid Arteries}

Carotid duplex ultrasound was performed using the Philips IU22 ultrasound system with 9-12 MHz linear array transducer in the NMRCC ultrasound laboratory. Atherosclerotic plaques were assessed in distal part of common carotid arteries (CCA) in anterior position, in bifurcation of CCA and in internal carotid arteries (ICA) from right and left side. Carotid plaques were defined as focal structures encroaching into the arterial lumen of at least $0.5 \mathrm{~mm}$ or $50 \%$ of surrounding intima-media thickness value or demonstrating a thickness greater than $1.5 \mathrm{~mm}$ as measured from the intima-lumen interface to the media-adventitia interface [12]. The carotid stenosis evaluation was made by using the ECST criteria [13]. The maximum percentage of arterial stenosis, summary percentage of arterial stenosis, and atherosclerotic plaques amount were evaluated. Atherosclerotic plaques amount was defined as the total amount of all plaques found in distal part of CCA, bifurcation of CCA and in ICA from right and left sides. Max\% of stenosis was defined as the maximum stenosis noted. Summary\% of stenosis was defined as the total amount of all stenosis found in distal part of CCA, bifurcation of CCA, and in ICA from right and left sides.

\subsection{Statistical Analysis}

Statistical analysis was performed with Statistica 6.0 (StatSoft Inc., Tulsa, OK, USA). Continuous variables are given as medians and interquartile ranges (lower and upper quartiles), Me(LQ-UQ). The characteristics between the groups were compared using the Mann-Whitney U test for continuous variables, and Fisher exact two-tailed and chi-squared tests for categorical data; $p$ value $<0.05$ was considered statistically significant. No Bonferroni corrections were made.

\section{Results}

\subsection{Genetic Test Results}

Likely pathogenic/pathogenic mutations, modifying factors, and protective factors were selected among other genetic variations while analyzing the results of NGS to prepare a clinical report for each patient. The information about the presence of these variants with their established clinical significance, associated max LDL-C/TC levels, data on cardiovascular disease (CVD), and a family history of death from myocardial infarction (MI) are given in Table 2. 
Table 2. Genetic tests results, disease phenotype and family history of death from myocardial infarction in 52 probands.

\begin{tabular}{|c|c|c|c|c|c|c|c|c|c|c|c|}
\hline \multirow{3}{*}{ Proband's ID } & \multicolumn{3}{|c|}{ Genetic Data } & \multicolumn{6}{|c|}{ Clinical Data } & \multicolumn{2}{|c|}{ Family History of Death from MI } \\
\hline & \multicolumn{2}{|c|}{ Mutations } & \multirow{2}{*}{ Modifying Factors $^{2}$} & \multirow{2}{*}{$\begin{array}{l}\text { Max LDL-C/TC } \\
\text { Levels, mmol/L } 10\end{array}$} & \multirow{2}{*}{$\begin{array}{l}\text { Lp(a) Levels, } \\
\text { mg/dL }\end{array}$} & \multirow{2}{*}{$\begin{array}{l}\text { HDL-C Levels, } \\
\text { mmol/L }\end{array}$} & \multirow{2}{*}{ Age, Years } & \multirow{2}{*}{ Sex } & \multirow{2}{*}{ Cardiovascular Disease } & \multirow{2}{*}{$\begin{array}{l}\text { First-Degree } \\
\text { Relatives }\end{array}$} & \multirow{2}{*}{$\begin{array}{c}\text { Second-Degree } \\
\text { Relatives }\end{array}$} \\
\hline & LDLR & АРОВ & & & & & & & & & \\
\hline 1 & - & Arg3527Gln & APOB (Glu2566Lys) ${ }^{3}$ & $6.19 / 8.60$ & 1.6 & 1.78 & 48 & $\mathrm{~F}$ & - & - & - \\
\hline 2 & Gly592Glu & - & - & $10.21 / 11.94^{9}$ & - & 1.35 & 65 & $\mathrm{~F}$ & PAD & - & - \\
\hline 4 & $\begin{array}{l}\text { c. } 940+\text { 3_940 + } \\
\text { 6delGAGT }\end{array}$ & - & $\begin{array}{l}\text { APOE (Cys130Arg) } \\
\text { APOA5 (Ser19Trp) }\end{array}$ & $9.17 / 11.89$ & 68.1 & 1.37 & 42 & $\mathrm{~F}$ & - & - & $\begin{array}{l}\text { Maternal uncle at } 45 \mathrm{y} \\
\text { maternal grandmothe } \\
\text { at } 59 \mathrm{yr} \text {, maternal uncle } \\
\text { SD at } 57 \mathrm{yr}\end{array}$ \\
\hline 6 & - & - & LPL (Asn318Ser) ${ }^{4}$ & $7.60 / 9.60$ & 7.5 & 1.2 & 56 & $\mathrm{~F}$ & PCI at 51yr & - & - \\
\hline 7 & Cys329Tyr & - & - & $10.00 / 12.00^{9}$ & 13.2 & 1.27 & 51 & $\mathrm{M}$ & PAD & - & - \\
\hline 8 & Gly592Glu & - & $\begin{array}{l}\text { APOE (Cys130Arg) }{ }^{3} \\
\text { LDLR (Val827Ile) }\end{array}$ & $11.50 / 14.00$ & 31 & 1.44 & 55 & $\mathrm{M}$ & $\begin{array}{c}\text { CABG at } 48 \mathrm{yr}, \text { PCI at } 50 \mathrm{yr} ; \\
\text { PAD }\end{array}$ & - & - \\
\hline 9 & - & - & & $6.06 / 8.00$ & 55.4 & 1 & 67 & $\mathrm{~F}$ & - & - & - \\
\hline 10 & - & - & LPA (Ile1891Met) ${ }^{5}$ & $8.43 / 11.11$ & 119.2 & 1.78 & 61 & $\mathrm{~F}$ & - & - & - \\
\hline 11 & - & - & - & $7.11 / 9.21$ & 106.4 & 1.09 & 42 & $\mathrm{M}$ & - & - & - \\
\hline 12 & - & - & - & $7.87 / 10.14$ & 11.7 & 1.38 & 42 & $\mathrm{M}$ & - & - & - \\
\hline 13 & - & - & - & $9.26 / 11.43$ & 54.9 & 1.35 & 45 & $\mathrm{M}$ & - & - & - \\
\hline 14 & - & - & APOE (Cys130Arg) ${ }^{3}$ & $9.4 / 11.50$ & 70.9 & 0.76 & 43 & $\mathrm{M}$ & - & - & - \\
\hline 16 & Cys352Arg & - & $\begin{array}{l}\text { APOE (Cys130Arg) } \\
\text { LPA (lle1891Met) }\end{array}$ & $8.59 / 10.13$ & 92.7 & 1 & 19 & $\mathrm{M}$ & - & - & $\begin{array}{l}\text { Paternal uncles at } 45 \mathrm{y}, \\
\text { and } 63 \mathrm{yr}\end{array}$ \\
\hline 17 & - & - & - & $10.85 / 14.25$ & 91.3 & 1.22 & 62 & $\mathrm{~F}$ & $\begin{array}{l}\text { CAD at 51yr, MI and CABG at } \\
62 \mathrm{yr}\end{array}$ & Mother at 38yr & - \\
\hline 18 & Pro106_Val395dup & - & $\begin{array}{l}\text { APOE (Cys130Arg) } \\
\text { APOA5 (Ser19Trp) }\end{array}$ & $8.17 / 9.94^{9}$ & 8.8 & 0.81 & 68 & M & $\mathrm{MI}$ at $51 \mathrm{yr}, \mathrm{CABG}$ at $61 \mathrm{yr}$ & Father at $67 \mathrm{yr}$ & - \\
\hline 19 & Val806Glyfs*11 & Arg3527Gln & $\begin{array}{l}\text { APOB (Glu2566Lys) }{ }^{3} \\
\text { PCSK } 9 \text { (Arg93Cys) }^{7}\end{array}$ & $9.36 / 12.00$ & 143.3 & 2.02 & 61 & $\mathrm{~F}$ & PCI at $48 \mathrm{yr} ; \mathrm{PAD}$ & Father at $69 \mathrm{yr}$ & Paternal uncle at $46 \mathrm{yr}$ \\
\hline 21 & c. $1846-3 \mathrm{~T}>\mathrm{G}$ & - & - & $8.94 / 10.57$ & 98.7 & 1.02 & 55 & M & $\begin{array}{l}\text { CAD at 46yr, CABG at 50yr; } \\
\text { PAD }\end{array}$ & - & Maternal uncle at $54 \mathrm{y}$ \\
\hline 22 & Ser586Pro & - & APOE (Cys130Arg $)^{3}$ & $9.68 / 12.00$ & 4.5 & 1.3 & 46 & M & $\begin{array}{l}\text { CAD at 36yr, MI and CABG at } \\
\text { 37yr; PAD }\end{array}$ & Mother at $58 \mathrm{yr}$ & $\begin{array}{c}\text { Two maternal uncles } \\
\text { before 40yr }\end{array}$ \\
\hline 23 & c. $2389+5 G>C$ & - & LPA (lle1891Met) ${ }^{5}$ & $13.11 / 15.67^{9}$ & 117.8 & 1.54 & 50 & $\mathrm{~F}$ & 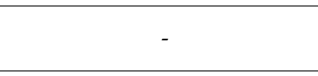 & - & $\begin{array}{c}\text { Maternal grandfather } \\
\text { at 52yr }\end{array}$ \\
\hline 24 & & - & APOE (Cys130Arg) ${ }^{3}$ & $7.76 / 9.96$ & 5 & 1.92 & 42 & $\mathrm{~F}$ & - & - & - \\
\hline 25 & $\begin{array}{l}\text { Ser177Leu, } \\
\text { Cys352Arg }\end{array}$ & - & & $17.63 / 19.00$ & 190.3 & 0.79 & 32 & $\mathrm{~F}$ & $\mathrm{CAD}$ at $20 \mathrm{yr}, \mathrm{MI}$ at $30 \mathrm{yr} ; \mathrm{PAD}$ & - & - \\
\hline 28 & $\begin{array}{l}\text { Cys329Tyr, } \\
\text { Gly592Glu }\end{array}$ & - & APOA5 (Ser19Trp) ${ }^{4}$ & $17.35 / 19.00$ & - & 0.98 & 39 & $\mathrm{~F}$ & CAD at $36 \mathrm{yr}$ & - & - \\
\hline 29 & $\begin{array}{l}\text { Arg416Trp, c.940+ } \\
\text { 3_940+6delGAGT }\end{array}$ & - & - & $15.15 / 17.25$ & 55.8 & 0.94 & 31 & $\mathrm{~F}$ & $\begin{array}{l}\text { MI at 15yr, CABG at 26yr; } \\
\text { PAD }\end{array}$ & - & - \\
\hline 30 & Pro220_Asp221del & - & - & $7.00 / 9.00$ & 6.5 & 0.95 & 46 & $\mathrm{M}$ & MI at $24 \mathrm{yr}, \mathrm{PCI}$ at $26 \mathrm{yr}$ & Mother at $62 \mathrm{yr}$ & - \\
\hline
\end{tabular}


Table 2. Cont.

\begin{tabular}{|c|c|c|c|c|c|c|c|c|c|c|c|}
\hline \multirow{3}{*}{ Proband's ID } & \multicolumn{3}{|c|}{ Genetic Data } & \multicolumn{6}{|c|}{ Clinical Data } & \multicolumn{2}{|c|}{ Family History of Death from MI } \\
\hline & \multicolumn{2}{|c|}{ Mutations } & \multirow{2}{*}{ Modifying Factors $^{2}$} & \multirow{2}{*}{$\begin{array}{l}\text { Max LDL-C/TC } \\
\text { Levels, mmol// } 10\end{array}$} & \multirow{2}{*}{$\begin{array}{l}\text { Lp(a) Levels, } \\
\text { mg/dL }\end{array}$} & \multirow{2}{*}{$\begin{array}{l}\text { HDL-C Levels, } \\
\mathrm{mmol} / \mathrm{L}\end{array}$} & \multirow{2}{*}{ Age, Years } & \multirow{2}{*}{ Sex } & \multirow{2}{*}{ Cardiovascular Disease } & \multirow{2}{*}{$\begin{array}{l}\text { First-Degree } \\
\text { Relatives }\end{array}$} & \multirow{2}{*}{$\begin{array}{c}\text { Second-Degree } \\
\text { Relatives }\end{array}$} \\
\hline & $L D L R$ & $A P O B$ & & & & & & & & & \\
\hline 33 & Gln739* & - & - & $7.00 / 9.10^{9}$ & 79.8 & 0.8 & 36 & $\mathrm{M}$ & MI and PCI at 36yr & - & - \\
\hline 34 & Gly119Valfs*12 & - & $\begin{array}{l}A P O E(\text { (Cys130Arg) } \\
\text { ABCA1 (Lys776Asn) }\end{array}$ & $11.00 / 13.00$ & 69.7 & 0.96 & 69 & $\mathrm{~F}$ & MI at 52yr, PCI at 66yr & $\begin{array}{l}\text { Mother at } 59 \mathrm{yr}, \\
\text { son SD at } 25 \mathrm{yr}\end{array}$ & $\begin{array}{c}\text { Maternal mother at } \\
64 \mathrm{yr} \text {, maternal aunt at } \\
69 \mathrm{yr}\end{array}$ \\
\hline 35 & - & - & - & $7.00 / 9.00$ & 3.3 & 0.85 & 47 & $\mathrm{M}$ & $\begin{array}{c}\text { CAD at } 39 \mathrm{yr}, \mathrm{PCI} \text { at } 40 \mathrm{yr}, \mathrm{MI} \\
\text { at } 46 \mathrm{yr} ; \mathrm{PAD}\end{array}$ & - & - \\
\hline 36 & Gly545Arg & - & - & $11.90 / 13.79$ & 25 & 1.01 & 53 & $\mathrm{~F}$ & CAD at $47 \mathrm{yr}$, PCI at $52 \mathrm{yr}$; PAD & - & - \\
\hline 37 & Tyr489Asn & - & $A P O A 5(\text { Ser19Trp })^{4}$ & $14.82 / 17.61$ & - & 1.1 & 37 & M & MI at $28 \mathrm{yr}$, PCI at $29 \mathrm{yr}$ & - & $\begin{array}{l}\text { Maternal aunt at 33yr } \\
\text { maternal grandfather } \\
\text { at 33yr }\end{array}$ \\
\hline 38 & $\begin{array}{l}\text { Glu714_Ile796del, } \\
\text { Trp443Arg }\end{array}$ & - & - & $21.00 / 23.00$ & 134 & 0.82 & 27 & $\mathrm{~F}$ & $\begin{array}{c}\text { MI at 21yr, CABG at 23yr; } \\
\text { PAD }\end{array}$ & - & $\begin{array}{c}\text { Maternal } \\
\text { grandmother at 60yr }\end{array}$ \\
\hline 39 & Lys581Gln & - & - & $11.18 / 12.80$ & - & 0.85 & 42 & $\mathrm{~F}$ & - & - & \\
\hline 40 & $\begin{array}{l}\text { Leu64_Pro105delinsSer, } \\
\text { Pro181Leu } \\
\end{array}$ & - & - & $8.83 / 10.83$ & - & 1.14 & 45 & $\mathrm{~F}$ & MI at $40 \mathrm{yr}, \mathrm{CABG}$ at $43 \mathrm{yr}$ & - & Paternal uncle at $57 \mathrm{yr}$ \\
\hline 41 & - & - & - & $6.70 / 9.38$ & 65.6 & 1.06 & 60 & $\mathrm{M}$ & $\begin{array}{l}\text { CAD at } 53 \mathrm{yr}, \mathrm{MI} \text { at } 58 \mathrm{yr}, \\
\text { CABG at } 59 \mathrm{yr} ; \text { PAD }\end{array}$ & - & - \\
\hline 42 & - & - & - & $7.53 / 9.68^{9}$ & 63 & 1.3 & 70 & $\mathrm{~F}$ & CAD at 48yr, MI at 51yr; PAD & Father at $61 \mathrm{yr}$ & - \\
\hline 43 & - & - & - & $6.08 / 9.14$ & 8.8 & 1.98 & 59 & $\mathrm{~F}$ & CAD at 56yr & - & - \\
\hline 44 & - & - & $L P A$ (Ile1891Met) $^{5}$ & $6.90 / 9.05$ & 105.7 & 1.64 & 61 & $\mathrm{~F}$ & & - & - \\
\hline 45 & Glu308Lys & - & $\begin{array}{l}\text { APOE }(\text { Cys130Arg) } \\
\text { LPA (lle1891Met) }{ }^{3}\end{array}$ & $8.30 / 10.30$ & 123.8 & 1.45 & 46 & $\mathrm{M}$ & $\mathrm{CAD}$ at $44 \mathrm{yr}, \mathrm{CABG}$ at $45 \mathrm{yr}$ & - & - \\
\hline 46 & - & - & $\begin{array}{l}\text { APOE }(\text { Cys130Arg) } \\
\text { LPA (Ile1891Met) }\end{array}$ & $8.00 / 10.00$ & 116.3 & 1.53 & 65 & $\mathrm{~F}$ & - & - & - \\
\hline 47 & Ser586Pro & - & LPA (Ile1891Met) ${ }^{5}$ & $7.84 / 9.75$ & 213 & 0.82 & 34 & $\mathrm{M}$ & $\begin{array}{l}\text { CAD at 31yr, MI and PCI at } \\
\text { 33yr }\end{array}$ & $\begin{array}{c}\text { Father SD at } \\
60 \mathrm{yr}\end{array}$ & - \\
\hline 48 & - & - & - & $5.55 / 8.00^{9}$ & 21.3 & 1.66 & 65 & M & MI and PCI at 59yr; PAD & $\begin{array}{c}\text { Father SD at } \\
48 \mathrm{yr}\end{array}$ & - \\
\hline 49 & - & - & APOE (Cys130Arg) ${ }^{3}$ & $5.75 / 7.95$ & 7 & 1.37 & 27 & $\mathrm{M}$ & $\mathrm{MI}$ and $\mathrm{PCI}$ at $21 \mathrm{yr}$ & - & $\begin{array}{c}\text { Maternal grandfather } \\
\text { at } 62 \mathrm{yr}\end{array}$ \\
\hline 50 & - & - & LIPC (c.-57_88del) ${ }^{4}$ & $9.00 / 11.00$ & 16.6 & 1.17 & 68 & $\mathrm{~F}$ & - & - & - \\
\hline 51 & Gly592Glu & - & - & $11.84 / 14.17$ & 7.5 & 1.13 & 59 & $\mathrm{~F}$ & - & - & - \\
\hline 52 & - & - & - & $14.67 / 17.00$ & 5.2 & 1.65 & 32 & $\mathrm{M}$ & - & - & - \\
\hline 53 & - & - & $\begin{array}{c}\text { APOE (Cys130Arg) } \\
\text { LPL (Ser474*) }\end{array}$ & $7.90 / 9.90$ & 2.9 & 1.22 & 56 & $\mathrm{M}$ & CAD at $54 \mathrm{yr}$ & - & - \\
\hline 54 & - & - & APOE (Cys130Arg) ${ }^{3}$ & $8.61 / 11.26$ & 2.6 & 0.68 & 46 & $\mathrm{M}$ & - & - & - \\
\hline
\end{tabular}


Table 2. Cont.

\begin{tabular}{|c|c|c|c|c|c|c|c|c|c|c|c|}
\hline \multirow{3}{*}{ Proband's ID } & \multicolumn{3}{|c|}{ Genetic Data } & \multicolumn{6}{|c|}{ Clinical Data } & \multicolumn{2}{|c|}{ Family History of Death from MI } \\
\hline & \multicolumn{2}{|c|}{ Mutations } & \multirow{2}{*}{ Modifying Factors $^{2}$} & \multirow{2}{*}{$\begin{array}{l}\text { Max LDL-C/TC } \\
\text { Levels, mmol/L } 10\end{array}$} & \multirow{2}{*}{$\begin{array}{l}\text { Lp(a) Levels, } \\
\text { mg/dL }\end{array}$} & \multirow{2}{*}{$\begin{array}{l}\text { HDL-C Levels, } \\
\mathrm{mmol} / \mathrm{L}\end{array}$} & \multirow{2}{*}{ Age, Years } & \multirow{2}{*}{ Sex } & \multirow{2}{*}{ Cardiovascular Disease } & \multirow{2}{*}{$\begin{array}{c}\text { First-Degree } \\
\text { Relatives }\end{array}$} & \multirow{2}{*}{$\begin{array}{c}\text { Second-Degree } \\
\text { Relatives }\end{array}$} \\
\hline & LDLR & $A P O B$ & & & & & & & & & \\
\hline 55 & - & - & APOA5 (Ser19Trp) ${ }^{4}$ & $6.1 / 8.64$ & 4 & 1.19 & 69 & $\mathrm{M}$ & - & - & - \\
\hline 56 & - & - & & $6.92 / 9.60$ & 9.9 & 2.24 & 55 & $\mathrm{~F}$ & CAD & - & - \\
\hline 57 & - & - & $A P O E(\text { Cys130Arg })^{3}$ & $5.98 / 8.00$ & 71.2 & 1.14 & 63 & $\mathrm{M}$ & $\begin{array}{l}\text { CAD at 38yr, MI and CABG at } \\
50 \mathrm{yr}\end{array}$ & Father at $48 \mathrm{yr}$ & - \\
\hline 58 & - & - & APOE $\left(\text { (Cys130Arg }{ }^{1}\right)^{3}$ & $8.00 / 10.10$ & 12.1 & 1.66 & 53 & $\mathrm{~F}$ & - & - & - \\
\hline 59 & - & - & $\begin{array}{l}\text { APOE (Cys130Arg) } \\
\text { LPL (Asn318Ser) }{ }^{4}\end{array}$ & $5.88 / 8.24$ & 2.9 & 1.23 & 44 & $\mathrm{~F}$ & - & - & - \\
\hline 60 & - & - & APOA5 (Ser19Trp) ${ }^{4}$ & $8.9 / 14.00^{9}$ & - & 1.4 & 44 & M & MI at $38 \mathrm{yr}$, PCI at 39yr; PAD & Father at $49 \mathrm{yr}$ & $\begin{array}{c}\text { Paternal grandfather } \\
\text { at } 53 \mathrm{yr}\end{array}$ \\
\hline
\end{tabular}

ABCA1, ATP-binding cassette sub-family A member 1; APOA5, Apolipoprotein A5; APOB, apolipoprotein B; APOE, apolipoprotein E; CABG, coronary artery bypass graft surgery;

$\mathrm{CAD}$, coronary artery disease; F, female; HDL-C, high-density lipoprotein cholesterol; ID, identifier; LDL-C, low-density lipoprotein cholesterol; LDLR, low density lipoprotein

receptor; LIPC, hepatic triacylglycerol lipase; LPA, apolipoprotein(a); Lp(a), lipoprotein(a); LPL, lipoprotein lipase; M, male; MI, myocardial infarction; PAD, peripheral arterial disease; PCI, percutaneous coronary intervention; PCSK9, proprotein convertase subtilisin/kexin type 9; SD, sudden death; TC, total cholesterol; yr, year. ${ }^{1} \mathrm{Homozygous}$. The unmarked variants are heterozygous. ${ }^{2}$ Polymorphisms previously associated with higher ${ }^{3}$ LDL-C, ${ }^{4}$ TG or ${ }^{5} \mathrm{Lp}(\mathrm{a})$ levels, or ${ }^{6}$ elevated ischemic heart disease risk. ${ }^{7}$ Polymorphism in the Japanese population, previously associated with low LDL-C levels and decreased risk of atherosclerotic CVD, and considered to be a protective factor. ${ }^{8}$ Rare variant of unknown clinical significance previously identified in patients with FH. ${ }^{9}$ On statins. ${ }^{10}$ LDL-C includes also Lp(a) cholesterol values. 
Pathogenic/likely pathogenic mutations considered to be responsible for monogenic FH were identified in 25 out of the 52 of probands (48\%): 24 with mutations in LDLR, 4 homozygous FH and 20 heterozygous $\mathrm{FH}(\mathrm{HeFH})$ carriers and 2 with a mutation in APOB (one of them also carried a pathogenic mutation in LDLR). There were 22 pathogenic/likely pathogenic mutations in LDLR: 12 missense, 3 frameshift, 3 deletions, 1 duplication and 3 splice-site mutations. Eight out of these 22 mutations in LDLR have not been previously described in the literature (Gly119Valfs*12, c.940 + 3_940 + 6delGAGT, Tyr489Asn, Lys581Gln, Ser586Pro, c.1846-3T > G, Glu714_Ile796del, c.2389 + 5G $>$ C). Only three (Pro220_Asp221del, Cys329Tyr, Trp443Arg) out of the 22 LDLR mutations have been previously reported in FH patients from Russia. The most frequent was a well-known mutation in LDLR, Gly592Glu, a founder mutation in a subpopulation in northwest Greece [14], also reported in the US, Europe and Latin America, which was found in four probands. The 22 mutations identified in the LDLR gene with their associated LDL-C levels, functional studies, and previous publications are listed in Table 4; additional information about predicted functional effects can be found in Tables S3-S5. Two patients carried a well-known pathogenic variant in the APOB gene, Arg3527Gln, which has been identified in combination with Glu2566Lys (rs1801696), a rare polymorphism in this gene [15]. There were no mutations in the proprotein convertase subtilisin/kexin type 9 gene.

All four patients with clinical picture of homozygous FH (maximum TC range of 17.3-23.0 mmol/L, maximum LDL-C range of 15.2-21.0 mmol/L) had two LDLR heterozygous mutations (Table 2).

All seven carriers who were heterozygous for the Ile1891Met polymorphism (rs3798220) in the lipoprotein(a) (LPA) gene [16] had elevated lipoprotein(a) [Lp(a)] levels, of 117.8 (105.7-123.8) mg/dL, ranging between 92.7 and $213.0 \mathrm{mg} / \mathrm{dL}$. Among non-carriers of Ile1891Met (or other variants in LPA to modify the phenotype), 16 patients had $\mathrm{Lp}(\mathrm{a})>50 \mathrm{mg} / \mathrm{dL}$, with median 71.1 (64.3-102.6) $\mathrm{mg} / \mathrm{dL}$. In addition, 12 probands were carriers of several polymorphisms (rs17602729, rs34526199, rs61752479) in the adenosine monophosphate deaminase 1 (AMPD1) gene [17], although they tolerated statins well. There were no dramatic adverse events on statin treatment; however, in 7 patients one statin was changed for another due to elevated transaminases, muscle pain or weakness ( 3 with and 4 without polymorphisms in AMPD1).

\subsection{Clinical Data Analysis for Heterozygous FH}

The comparison of clinical characteristics of mutation-positive and mutation-negative patients with $\mathrm{HeFH}$ is given in Table 5. Among patients with clinically established $\mathrm{HeFH}$, the maximum TC and LDL-C levels were higher in those with positive than in those with negative genetic test results ( $p=0.01$ and $p=0.0004$, respectively). However, current untreated median LDL-C levels were quite similar $(8.9 \mathrm{mmol} / \mathrm{L}$ in positive and $7.9 \mathrm{mmol} / \mathrm{L}$ in mutation-negative cases, $p=0.04)$, as well as the number of patients who had suffered from MI, which did not significantly differ between the groups. Although mutation-negative patients had highly elevated TC and LDL-C levels, only a half of them had a positive family history of hypercholesterolemia in first-degree relatives. The mutation-positive patients had more severe atherosclerotic lesions in carotid arteries and a higher percentage of premature peripheral artery disease $(p=0.03)$, see Figure 1 . 
Table 3. List of 22 mutations identified in the LDLR gene with their associated low-density lipoprotein cholesterol (LDL-C)/total cholesterol (TC) levels, functional studies and previous publications.

\begin{tabular}{|c|c|c|c|c|c|c|c|c|c|c|c|c|c|}
\hline $\mathbf{N}$ & LDLR Variant & Exon/Intron & c.DNA & $\begin{array}{l}\text { Number } \\
\text { of Pts }\end{array}$ & $\begin{array}{c}\text { Associated }{ }^{1} \text { Max } \\
\text { LDL-C/TC Levels, } \\
\text { mmol/1 }\end{array}$ & $\begin{array}{c}\text { Previously } \\
\text { Published in } \\
\text { Publications }\end{array}$ & $\begin{array}{c}\text { ExAC } \\
\text { Database } \\
\text { Frequency }\end{array}$ & $\begin{array}{l}\text { GnomAD } \\
\text { Allele } \\
\text { Frequencies }\end{array}$ & $\begin{array}{l}\text { Familial } \\
\text { Cosegregation }\end{array}$ & $\begin{array}{l}\text { Functional } \\
\text { Studies }\end{array}$ & $\begin{array}{c}\text { Number of } \\
\text { Carriers/Families }\end{array}$ & $\begin{array}{l}\text { Countries } \\
\text { (Previously } \\
\text { Published) }\end{array}$ & Pathogenicity \\
\hline 1 & Leu64_Pro105delinsSer & Ex3 & c.191_313del & 1 & - & 6 & 0 & 0 & - & + & NA & $\begin{array}{l}\text { France, the } \\
\text { Netherlands }\end{array}$ & +++ \\
\hline 2 & Pro106_Val395dup & DupEx4-8 & c. ${ }^{*} 11173514 \_* 11173515$ & 1 & $8.17 / 9.94^{2,3}$ & 3 & 0 & 0 & - & - & NA & $\begin{array}{l}\text { Poland, the } \\
\text { Czech } \\
\text { Republic, the } \\
\text { Netherlands }\end{array}$ & +++ \\
\hline 3 & Gly119Valfs*12 & Ex4 & c.355_356insTTCC & 1 & $11.00 / 13.00^{2,3}$ & 0 & 0 & 0 & - & - & 0 & - & +++ \\
\hline 4 & $\begin{array}{l}\text { Ser177Leu, FH Puerto } \\
\text { Rico }\end{array}$ & Ex4 & c. $530 \mathrm{C}>\mathrm{T}$ & 1 & - & 36 & 1/121078 & $4 / 251308$ & + & + & $59 / 33$ & $\begin{array}{l}\text { Europe, Latin } \\
\text { America, USA }\end{array}$ & +++ \\
\hline 5 & Pro181Leu & Ex4 ${ }^{4}$ & c. $542 \mathrm{C}>\mathrm{T}$ & 1 & - & 1 & 0 & $2 / 251308$ & - & + & $1 / 1$ & Brazil & + \\
\hline 6 & Pro220_Asp221del & Ex4 & c.658_663delCCCGAC & 1 & $7.0 / 9.0$ & 1 & 0 & 0 & - & + & $1 / 1$ & Russia & +++ \\
\hline 7 & Glu308Lys & Ex6 & c. $922 \mathrm{G}>\mathrm{A}$ & 1 & $8.3 / 10.3^{2}$ & 3 & 0 & 0 & - & - & NA & $\begin{array}{l}\text { Poland, the } \\
\text { Netherlands }\end{array}$ & ++ \\
\hline 8 & $\begin{array}{c}\text { c.940 + 3_940 + } \\
\text { 6delGAGT }\end{array}$ & Int6 & $\begin{array}{c}\text { c.940+ 3_940 + } \\
\text { 6delGAGT } \\
\end{array}$ & 2 & $9.17 / 11.89^{2}$ & 0 & 0 & 0 & - & - & 0 & - & + \\
\hline 9 & Cys329Tyr & $\operatorname{Ex} 7^{4}$ & c. $986 \mathrm{G}>\mathrm{A}$ & 2 & $10.00 / 12.00^{3}$ & 15 & 3/120592 & $7 / 282402$ & + & - & $53 / 38$ & $\begin{array}{l}\text { Asia (Taiwan, } \\
\text { Philippines, } \\
\text { China) and } \\
\text { Russia }\end{array}$ & +++ \\
\hline 10 & Cys352Arg & $\mathrm{Ex} \times 7^{4}$ & c. $1054 \mathrm{~T}>\mathrm{C}$ & 2 & $8.59 / 10.13^{2}$ & 4 & 0 & 0 & - & - & $\mathrm{NA}$ & Austria & ++ \\
\hline 11 & Arg416Trp & Ex9 & c. $1246 \mathrm{C}>\mathrm{T}$ & 1 & - & 42 & 3/120616 & $6 / 251158$ & + & + & $\mathrm{NA}$ & $\begin{array}{c}\text { Europe (Spain, } \\
\text { Norway, the } \\
\text { UK, Germany, } \\
\text { Austria, Czech } \\
\text { Republic), } \\
\text { Canada, Asia } \\
\text { (Japan, } \\
\text { Taiwan) }\end{array}$ & +++ \\
\hline 12 & Trp443Arg & $\operatorname{Ex} 9^{4}$ & c.1327 T > C & 1 & - & 1 & $1 / 120926$ & $1 / 251246$ & - & - & $2 / 2$ & Russia & ++ \\
\hline 13 & Tyr489Asn & $E \times 10^{4}$ & c. $1465 \mathrm{~T}>\mathrm{A}$ & 1 & $14.82 / 17.61$ & 0 & 0 & 0 & - & - & 0 & - & + \\
\hline 14 & Gly545Arg & Ex11 ${ }^{4}$ & c. $1633 \mathrm{G}>\mathrm{A}$ & 1 & $11.9 / 13.79$ & 5 & 0 & 0 & - & - & $5 / 5$ & $\begin{array}{c}\text { Brazil, France, } \\
\text { Korea }\end{array}$ & ++ \\
\hline 15 & Lys581Gln & Ex12 ${ }^{4}$ & c. $1741 \mathrm{~A}>\mathrm{C}$ & 1 & $11.18 / 12.80$ & 0 & 0 & 0 & - & - & 0 & - & + \\
\hline 16 & Ser586Pro & Ex12 & c.1756 T > C & 2 & $\begin{array}{c}9.68 / 12.00^{2} \\
7.84 / 9.39\end{array}$ & 0 & 0 & 0 & - & - & 0 & - & + \\
\hline
\end{tabular}


Table 4. List of 22 mutations identified in the LDLR gene with their associated low-density lipoprotein cholesterol (LDL-C)/total cholesterol (TC) levels, functional studies and previous publications.

\begin{tabular}{|c|c|c|c|c|c|c|c|c|c|c|c|c|c|}
\hline $\mathbf{N}$ & LDLR Variant & Exon/Intron & c.DNA & $\begin{array}{c}\text { Number } \\
\text { of Pts }\end{array}$ & $\begin{array}{c}\text { Associated }^{1} \text { Max } \\
\text { LDL-C/TC Levels, } \\
\text { mmol/1 }\end{array}$ & $\begin{array}{c}\text { Previously } \\
\text { Published in } \\
\text { Publications }\end{array}$ & $\begin{array}{c}\text { ExAC } \\
\text { Database } \\
\text { Frequency }\end{array}$ & $\begin{array}{l}\text { GnomAD } \\
\text { Allele } \\
\text { Frequencies }\end{array}$ & $\begin{array}{l}\text { Familial } \\
\text { Cosegregation }\end{array}$ & $\begin{array}{l}\text { Functional } \\
\text { Studies }\end{array}$ & $\begin{array}{c}\text { Number of } \\
\text { Carriers/Families }\end{array}$ & $\begin{array}{l}\text { Countries } \\
\text { (Previously } \\
\text { Published) }\end{array}$ & Pathogenicity \\
\hline 17 & $\begin{array}{l}\text { Gly592Glu, FH Sicily, } \\
\text { FH Foggia-1, FH } \\
\text { Naples4 }\end{array}$ & Ex12 & c. $1775 \mathrm{G}>\mathrm{A}$ & 4 & $\begin{array}{c}10.21 / 11.94^{3} \\
11.50 / 14.00^{2} \\
11.84 / 14.17\end{array}$ & 41 & 6/121408 & $16 / 282866$ & + & + & $31 / 27$ & $\begin{array}{c}\text { The most } \\
\text { common cause } \\
\text { of FH in } \\
\text { north-western } \\
\text { Greece (34\% of } \\
\text { cases); USA, } \\
\text { Europe, Latin } \\
\text { America }\end{array}$ & +++ \\
\hline 18 & c.1846-3T > G & Int12 & c.1846-3T > G & 1 & $8.94 / 10.57$ & 0 & 0 & 0 & - & - & 0 & - & + \\
\hline 19 & Glu714_Ile796del & Int14-Int16 & 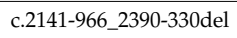 & 1 & - & 0 & 0 & 0 & + & + & $11 / 2$ & Japan, Brazil & +++ \\
\hline 20 & Gln739* & Ex15 & c. $2215 \mathrm{C}>\mathrm{T}$ & 1 & $7.0 / 9.1^{3}$ & 9 & 0 & 0 & - & - & $5 / 5$ & $\begin{array}{l}\text { Italy, Mexico, } \\
\text { Asia (Japan, } \\
\text { Taiwan) }\end{array}$ & +++ \\
\hline 21 & c. $2389+5 G>C$ & Int16 ${ }^{4}$ & c. $2389+5 G>C$ & 1 & $13.11 / 15.67^{3}$ & 0 & 0 & 0 & - & - & 0 & - & ++ \\
\hline 22 & Val806Glyfs" 11 & Ex17 & c.2416_2417insG & 1 & - & 17 & $5 / 121318$ & $6 / 251326$ & + & - & 20/10 & $\begin{array}{l}\text { USA, Europe, } \\
\text { Middle East, } \\
\text { Latin America, } \\
\text { Japan }\end{array}$ & +++ \\
\hline
\end{tabular}

c.DNA, coding DNA; ExAC, Exome Aggregation Consortium; FH, familial hypercholesterolemia; gnomAD, Genome Aggregation Database (v2.1.1 version); LDL-C, low-density lipoprotein cholesterol; LDLR, low density lipoprotein receptor; N, mutation number; NA, not available; pts, patients; TC, total cholesterol; +++, Pathogenic; ++, Very likely pathogenic; +, Likely pathogenic. ${ }^{1}$ The max LDL-C/TC levels in a single-mutation heterozygous carriers (carriers of more than one mutation in LDLR are excluded). ${ }^{2}$ Heterozygous for ApoE4. ${ }^{3}$ On statins.

Other variants in the same position were previously reported in association with FH. 
Table 5. Clinical characteristics of patients with heterozygous familial hypercholesterolemia.

\begin{tabular}{|c|c|c|c|c|}
\hline \multirow{2}{*}{\multicolumn{2}{|c|}{ Clinical Characteristics }} & \multicolumn{2}{|c|}{ Mutation } & \multirow{2}{*}{$p$} \\
\hline & & Positive $(n=21)$ & Negative $(n=27)$ & \\
\hline \multicolumn{5}{|c|}{ Clinical data } \\
\hline \multicolumn{2}{|c|}{ Men/Women } & $11 / 10$ & $14 / 13$ & NS \\
\hline \multicolumn{2}{|c|}{ Mean age, years } & $48(42-55)$ & $56(44-63)$ & NS \\
\hline \multicolumn{2}{|c|}{ BMI, $\mathrm{kg} / \mathrm{m}^{2}$} & $27.4(24.1-30.8)$ & $28.4(24.5-31.1)$ & NS \\
\hline \multicolumn{2}{|c|}{ Smokers: Present/Gave up } & $29 \% / 24 \%$ & $22 \% / 33 \%$ & NS \\
\hline \multicolumn{2}{|c|}{ Arterial hypertension } & $67 \%$ & $56 \%$ & NS \\
\hline \multicolumn{5}{|c|}{ Family history } \\
\hline \multicolumn{2}{|c|}{ Premature CAD } & $52 \%$ & $63 \%$ & NS \\
\hline \multicolumn{2}{|c|}{ Tendon xanthomas } & $14 \%$ & $0 \%$ & 0.08 \\
\hline \multicolumn{2}{|c|}{ Hypercholesterolemia } & $86 \%$ & $48 \%$ & 0.007 \\
\hline \multicolumn{5}{|c|}{ Personal history } \\
\hline \multicolumn{2}{|c|}{ Tendon xanthomas } & $33 \%$ & $11 \%$ & 0.06 \\
\hline \multicolumn{2}{|c|}{ Premature CAD/CAD } & $62 \% / 62 \%$ & $37 \% / 44 \%$ & $0.08 / \mathrm{NS}$ \\
\hline \multicolumn{2}{|c|}{ Myocardial infarction } & $38 \%$ & $33 \%$ & NS \\
\hline \multicolumn{2}{|c|}{ Age of MI } & $36.5(30.5-45.5)$ & $50.5(42.0-58.5)$ & NS \\
\hline \multicolumn{2}{|c|}{ PCI } & $38 \%$ & $19 \%$ & NS \\
\hline \multicolumn{2}{|c|}{ CABG } & $29 \%$ & $11 \%$ & NS \\
\hline \multicolumn{2}{|c|}{ Premature PAD/PAD } & $33 \% / 33 \%$ & $7 \% / 19 \%$ & $0.03 / \mathrm{NS}$ \\
\hline \multicolumn{2}{|c|}{ Ischemic stroke } & $5 \%$ & $4 \%$ & NS \\
\hline \multicolumn{5}{|c|}{ Laboratory data } \\
\hline \multicolumn{2}{|c|}{ Max TC, mmol/L } & $11.9(10.1-13.0)$ & $9.6(9.0-11.0)$ & 0.01 \\
\hline \multicolumn{2}{|c|}{ Max LDL-C, mmol/L } & $9.4(8.3-11.2)$ & $7.6(6.6-8.6)$ & 0.0004 \\
\hline \multirow{4}{*}{$\begin{array}{c}\text { Current lipid levels, } \\
\mathrm{mmol} / \mathrm{L}\end{array}$} & TC & $10.3(9.1-12.0)$ & $9.7(8.1-11.1)$ & NS \\
\hline & LDL-C & $8.6(7.0-10.2)$ & $7.6(6.1-8.6)$ & 0.07 \\
\hline & HDL-C & $1.1(1.0-1.4)$ & $1.3(1.1-1.7)$ & NS \\
\hline & TG & $1.6(1.2-2.3)$ & $1.9(1.7-2.1)$ & NS \\
\hline \multicolumn{2}{|c|}{ Current LDL-C without treatment, $\mathrm{mmol} / \mathrm{L}$} & $\begin{array}{l}8.9(8.2-11.5) \\
\quad(n=12)\end{array}$ & $\begin{array}{c}7.9(6.7-8.6) \\
(n=21)\end{array}$ & 0.04 \\
\hline \multicolumn{2}{|c|}{$\mathrm{Lp}(\mathrm{a}), \mathrm{mg} / \mathrm{dL}$} & $\begin{array}{l}68.1(8.8-98.7) \\
\quad(n=17)\end{array}$ & $\begin{array}{l}14.35(5.2-70.9) \\
\quad(n=26)\end{array}$ & NS \\
\hline \multicolumn{5}{|c|}{ Duplex ultrasound of carotid arteries data } \\
\hline \multicolumn{2}{|c|}{ Max $\%$ of stenosis ${ }^{1}$} & $35(25-45)$ & $25(20-35)$ & 0.04 \\
\hline Summary\% & & $145(50-210)$ & $60(20-125)$ & 0.01 \\
\hline Atherosclerotic $p$ & nount ${ }^{3}$ & $5(2-6)$ & $3(1-4)$ & 0.003 \\
\hline
\end{tabular}

BMI, body mass index; CABG, coronary artery bypass graft surgery; CAD, coronary artery disease; HDL-C, high-density lipoprotein cholesterol; LDL-C, low-density lipoprotein cholesterol; Lp(a), lipoprotein(a); MI, myocardial infarction; PAD, peripheral arterial disease; PCI, percutaneous coronary intervention; TC, total cholesterol; TG, triglyceride. NS, $p \geq 0.1 .{ }^{1}$ One maximal stenosis is chosen. ${ }^{2}$ Total amount of all stenosis found in distal part of common carotid artery, bifurcation of common carotid artery and in internal carotid artery from right and left sides. ${ }^{3}$ Total amount of all plaques found in distal part of common carotid artery, bifurcation of common carotid artery and in internal carotid artery from right and left sides. 


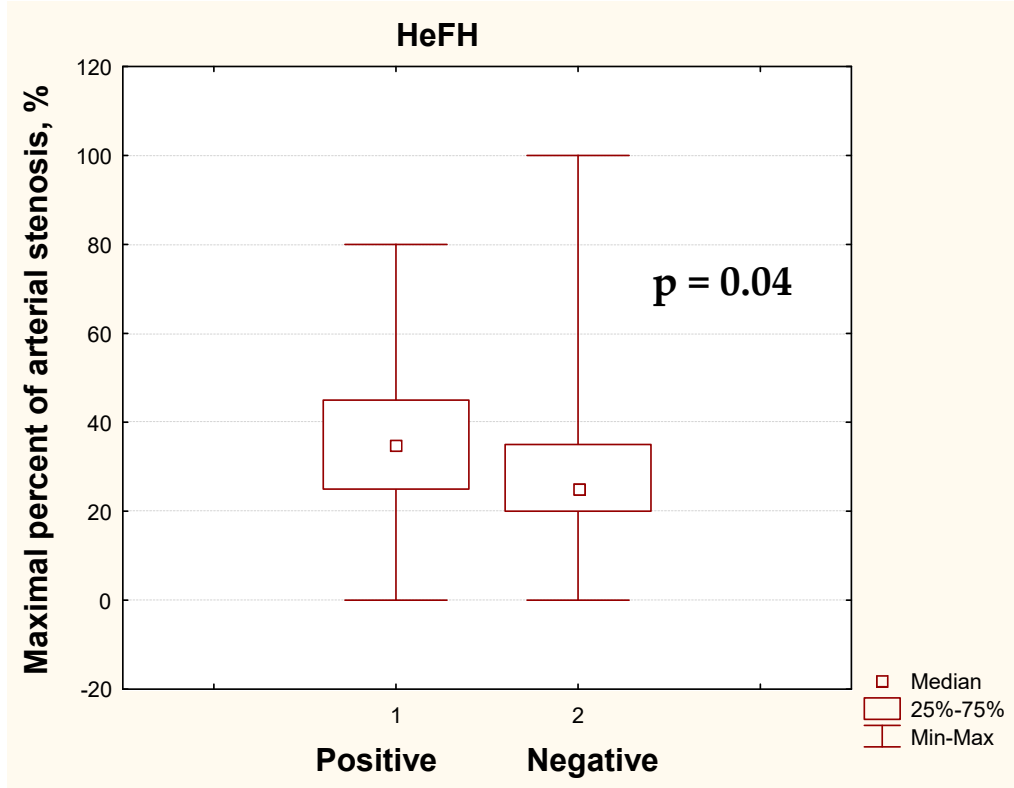

(a)

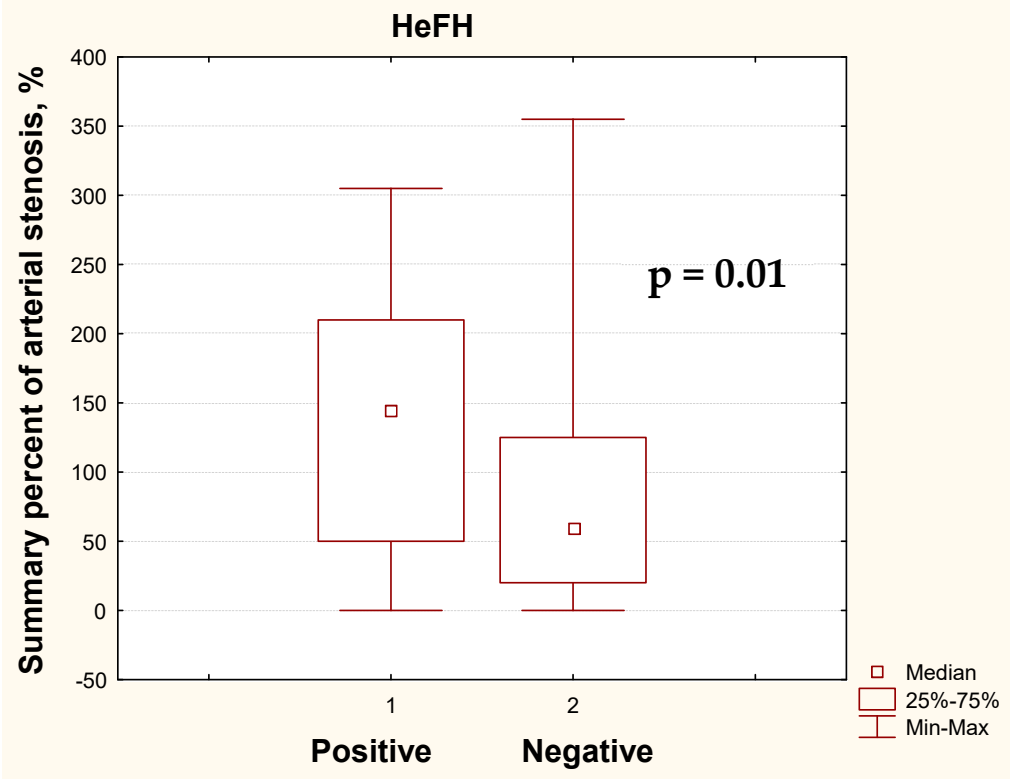

(b)

Figure 1. Cont. 


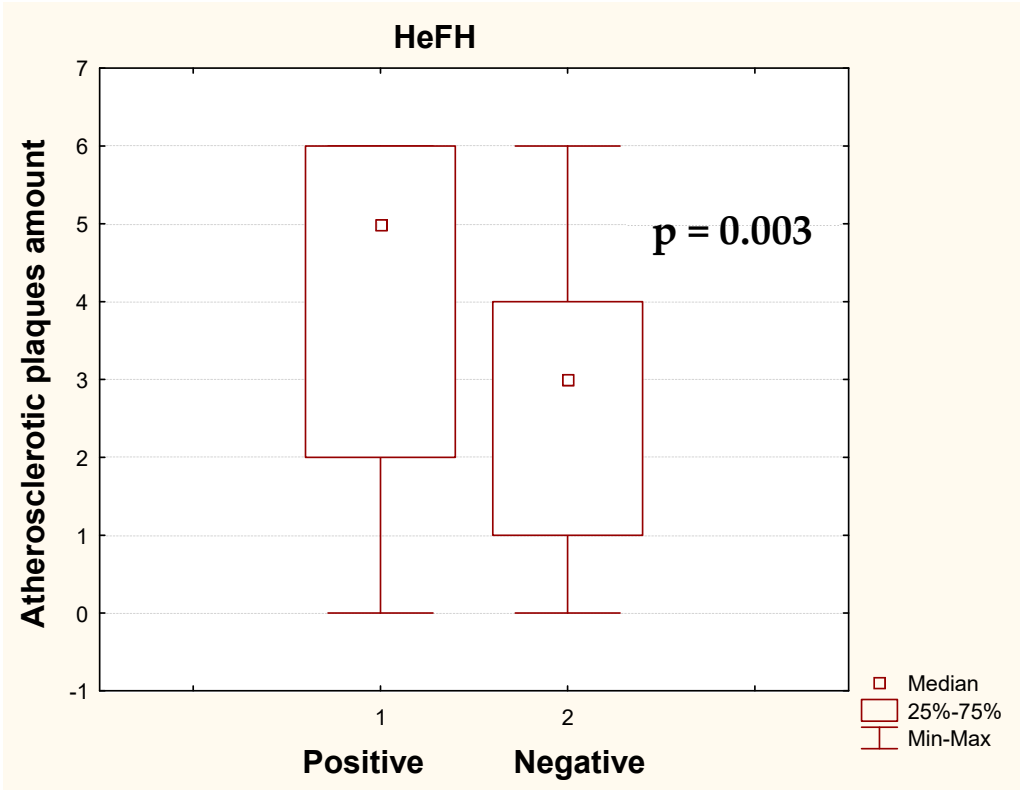

(c)

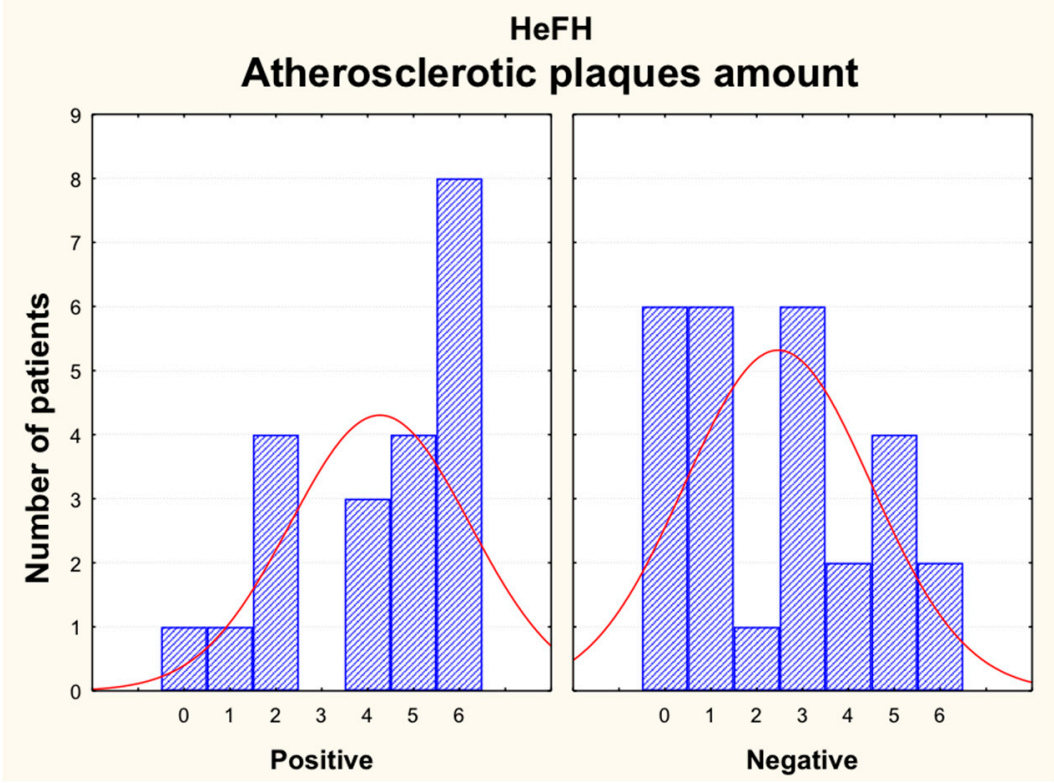

(d)

Figure 1. The carotid duplex ultrasound data in mutation-positive (1) and mutation-negative (2) patients with clinically established heterozygous familial hypercholesterolemia ( $\mathrm{HeFH})$ : (a) maximal percent of arterial stenosis; (b) summary percent of arterial stenosis; (c,d) atherosclerotic plaques amount.

\section{Discussion}

With the development of new technologies, the impact of genetic studies for clinical practice is being widely evaluated. It became clear that the genetic cause of the disease is not always identifiable in patients with a clinical diagnosis of FH. On the other hand, in some patients, this genetic cause does not involve only one gene (polygenic cause of the disease, determined by the accumulation of common variants, single nucleotide polymorphisms [SNPs], which increase LDL-C) [18]. In our study, the disease-causing mutations, mostly in LDLR, have been identified only in $48 \%$ of patients with clinical diagnosis of $\mathrm{FH}$, which is consistent with data received from other countries. At the same time 
there was a difference among mutation-positive and mutation-negative patients in clinical presentation of disease (Table 5).

There are convincing data confirming that the presence of a FH-related mutation leads to prolonged exposure to LDL-C and, therefore, significantly elevated cardiovascular (CV) risk [19]. The preclinical atherosclerosis, assessed by carotid intima-media thickness measurements and the coronary artery calcium score, was found to be more severe in patients with monogenic FH [20]. It was shown that among individuals with LDL-C $\geq 4.9 \mathrm{mmol} / \mathrm{L}$, those with pathogenic mutations identified by sequencing three main FH genes (LDLR, APOB and PCSK9) have approximately 3.7-fold higher risk for coronary artery disease [21]. In genotyped HeFH patients registered in the Norwegian Cause of Death Registry during 1992-2013, CVD was the most common cause of death, with mean age of 64.5 years [22]. However, the number of patients in our study who had suffered from MI did not significantly differ depending on a presence of pathogenic mutations, for all patients with heterozygous $\mathrm{FH}$, as well as when calculated for 50-year-olds or younger $(p=0.3)$. These data support the findings that other $\mathrm{CV}$ risk factors, besides $\mathrm{FH}$, add significant impact in possibility of MI development [23]. The independent association of age, male sex, increased body mass index (BMI), hypertension, type 2 diabetes mellitus, previous use of tobacco and $\mathrm{Lp}(\mathrm{a})>50 \mathrm{mg} / \mathrm{dL}$ with atherosclerotic CVD was confirmed in patients from the SAFEHEART Registry (Spanish Familial Hypercholesterolemia Cohort Study) with molecularly defined HeFH [24]. Recently, several risk scores (the Montreal-FH-SCORE, the SAFEHEART-RE risk equation) based on simple clinical predictors were found appropriate for CV risk assessment in patients with a genetically confirmed HeFH $[25,26]$ Similarities in parameters such as arterial hypertension, smoking habit, BMI, HDL-C levels, age and gender were present between the groups of mutation-positive and mutation-negative patients in our study (Table S6).

The phenotype may also be modified by common variants associated with high LDL-C levels and CVD risk, which sometimes brings a visible variability within one family [27]. Among mutationnegative patients in our study, there were no significant differences in lipid levels depending on the presence of the APOE4 allele, showing the importance of particular combinations of SNPs for a prominent hypercholesterolemia.

\section{Conclusions}

Monogenic FH has been genetically confirmed in about half of the patients with definite or probable diagnosis of FH in our study, and the importance of a genetic study to determine the pathogenic cause of FH can be emphasized. Carriers of pathogenic mutations are more likely to have severe atherosclerotic lesions and tendon xanthomas due to prolonged exposure to LDL-C. Moreover, the autosomal-dominant type of inheritance predicts the signs of disease in relatives, making genetic cascade family screening reasonable for a timely identification of individuals at high CV risk [28]. At the same time, the importance of other $\mathrm{CV}$ risk factors should not be underestimated in patients with FH.

Supplementary Materials: The following are available online at http://www.mdpi.com/2308-3425/7/2/16/s1, Table S1. List of 63 evaluated genes related to dyslipidemia and premature atherosclerosis with associated phenotypes. Table S2: Customized classification scheme based on the recommendations of the ACMG used for establishing the pathogenicity of the identified variants, Table S3: List of mutations identified in the LDLR gene with nomenclature and their predicted functional effects on the protein, Table S4: Mutation effect prediction algorithms-Missense mutations in LDLR, Table S5: Mutation effect prediction algorithms-Splice-site mutations in LDLR, Table S6: Cardiovascular risk factors and presence of myocardial infarction in patients with heterozygous familial hypercholesterolemia.

Author Contributions: Conceptualization, A.E.S., I.V.S. and M.V.E.; methodology, A.E.S., I.V.S. and M.V.E.; formal analysis, A.E.S.; investigation, A.E.S., D.G.-G., L.M., A.B.P. and D.N.N.; writing-original draft preparation, A.E.S.; writing-review and editing, A.E.S., I.V.S., M.V.E., D.G.-G. and L.M.; supervision, I.V.S. and M.V.E. All authors have read and agreed to the published version of the manuscript.

Funding: The genetic study was partly sponsored by the Russian National Atherosclerosis Society, IAS and Pfizer Independent Grant for Learning \& Change (ID 11532493), Amgen, AstraZeneca, INVITRO Laboratory. 
Acknowledgments: The study was performed with the organizational support of the Russian National Atherosclerosis Society and National Medical Research Center of Cardiology, Moscow, Russia. We would like to thank Natalia Sonicheva for organizational support, Radek Suchác for helping with grammar and style editing and other members of the Health in Code company team for all the contributions they made to this project and also the administration and all the involved employees of the National Medical Research Center of Cardiology in Moscow, Russia.

Conflicts of Interest: The authors declare no conflict of interest. The funders had no role in the design of the study; in the collection, analyses or interpretation of data; in the writing of the manuscript or in the decision to publish the results.

\section{References}

1. Ezhov, M.V.; Sergienko, I.V.; Duplyakov, D.V.; Abashina, O.E.; Kachkovsky, M.A.; Shaposhnik, I.I.; Genkel, V.V.; Muzalevskaya, M.V. Results of the Russian research program on the diagnosis and treatment of patients with familial hypercholesterolaemia. High prevalence, low awareness, poor adherence. J. Atheroscler. Dyslipidaemias 2017, 2, 5-15.

2. Ershova, A.I.; Meshkov, A.N.; Bazhan, S.S.; Storozhok, M.A.; Efanov, A.Y.; Medvedeva, I.V.; Indukaeva, E.V.; Danilchenko, Y.; Kuzmina, O.K.; Barbarash, O.L.; et al. The prevalence of familial hypercholesterolemia in the West Siberian region of the Russian Federation: A substudy of the ESSE-RF. PLoS ONE 2017, 12, e0181148. [CrossRef] [PubMed]

3. Nordestgaard, B.G.; Chapman, M.J.; Humphries, S.E.; Ginsberg, H.N.; Masana, L.; Descamps, O.S.; Wiegman, A. European Atherosclerosis Society Consensus Panel. Familial hypercholesterolaemia is underdiagnosed and undertreated in the general population: Guidance for clinicians to prevent coronary heart disease: Consensus statement of the European Atherosclerosis Society. Eur. Heart J. 2013, 34, 3478-3490. [CrossRef] [PubMed]

4. De Ferranti, S.D.; Rodday, A.M.; Mendelson, M.; Wong, J.B.; Leslie, L.K.; Sheldrick, R.C. Prevalence of Familial Hypercholesterolemia in the 1999 to 2012 United States National Health and Nutrition Examination Surveys (NHANES). Circulation 2016, 133, 1067-1072. [CrossRef] [PubMed]

5. Schmidt, N.; Schmidt, B.; Dressel, A.; Gergei, I.; Klotsche, J.; Pieper, L.; Scharnagl, H.; Kleber, M.; März, W.; Lehnert, H.; et al. Familial hypercholesterolemia in primary care in Germany. Diabetes and cardiovascular risk evaluation: Targets and Essential Data for Commitment of Treatment (DETECT) study. Atherosclerosis 2017, 266, 24-30. [CrossRef] [PubMed]

6. Benn, M.; Watts, G.F.; Tybjærg-Hansen, A.; Nordestgaard, B.G. Mutations causative of familial hypercholesterolaemia: Screening of 98098 individuals from the Copenhagen General Population Study estimated a prevalence of 1 in 217. Eur. Hear. J. 2016, 37, 1384-1394. [CrossRef]

7. Safarova, M.S.; Sergienko, I.V.; Ezhov, M.V.; Semenova, A.E.; Kachkovskiy, M.A.; Shaposhnik, I.I.; on behalf of the RuFH Investigators. Russian research program for early diagnosis and treatment of familial hypercholesterolaemia: Rationale and Design of the Russian FH Registry (RuFH). J. Atheroscler. Dyslipidaemias 2014, 3, 7-15.

8. Santos, R.D.; Gidding, S.S.; Hegele, R.A.; Cuchel, M.A.; Barter, P.J.; Watts, G.F.; Baum, S.J.; Catapano, A.L.; Chapman, M.J.; Defesche, J.C.; et al. Defining severe familial hypercholesterolaemia and the implications for clinical management: A consensus statement from the International Atherosclerosis Society Severe Familial Hypercholesterolemia Panel. Lancet Diabetes Endocrinol. 2016, 4, 850-861. [CrossRef]

9. Richards, S.; on behalf of the ACMG Laboratory Quality Assurance Committee; Aziz, N.; Bale, S.; Bick, D.; Das, S.; Gastier-Foster, J.; Grody, W.W.; Hegde, M.; Lyon, E.; et al. Standards and guidelines for the interpretation of sequence variants: A joint consensus recommendation of the American College of Medical Genetics and Genomics and the Association for Molecular Pathology. Genet. Med. 2015, 17, 405-423. [CrossRef]

10. Friedewald, W.T.; Levy, R.I.; Fredrickson, D.S. Estimation of the Concentration of Low-Density Lipoprotein Cholesterol in Plasma, Without Use of the Preparative Ultracentrifuge. Clin. Chem. 1972, 18, 499-502. [CrossRef]

11. Afanasieva, O.I.; Adamova, I.Y.; Benevolenskaya, G.F.; Pokrovsky, S.N. Immuno-enzyme assay for lipoprotein(a) measurement. Bull. Exp. Biol. Med. 1995, 4, 398-401.

12. Touboul, P.-J.; Hennerici, M.; Meairs, S.; Adams, H.; Amarenco, P.; Bornstein, N.; Csiba, L.; Desvarieux, M.; Ebrahim, S.; Hernandez, R.H.; et al. Mannheim Carotid Intima-Media Thickness and Plaque Consensus (2004-2006-2011). Cerebrovasc. Dis. 2012, 34, 290-296. [CrossRef] [PubMed] 
13. European Carotid Surgery Trialists' Collaborative Group. Randomised trial of endarterectomy for recently symptomatic carotid stenosis: Final results of the MRC European Carotid Surgery Trial (ECST). Lancet 1998, 351, 1379-1387. [CrossRef]

14. Diakou, M.; Miltiadous, G.; Xenophontos, S.L.; Manoli, P.; Cariolou, M.A.; Elisaf, M. Spectrum of LDLR gene mutations, including a novel mutation causing familial hypercholesterolaemia, in North-western Greece. Eur. J. Int. Med. 2011, 22, e55-e59. [CrossRef]

15. Huijgen, R.; Sjouke, B.; Vis, K.; De Randamie, J.S.; Defesche, J.C.; Kastelein, J.J.; Hovingh, G.K.; Fouchier, S.W. Genetic variation in APOB, PCSK9, and ANGPTL3 in carriers of pathogenic autosomal dominant hypercholesterolemic mutations with unexpected low LDL-Cl Levels. Hum. Mutat. 2011, 33, 448-455. [CrossRef]

16. Clarke, R.; Peden, J.F.; Hopewell, J.C.; Kyriakou, T.; Goel, A.; Heath, S.; Parish, S.; Barlera, S.; Franzosi, M.G.; Rust, S.; et al. Genetic Variants Associated with Lp(a) Lipoprotein Level and Coronary Disease. N. Engl. J. Med. 2009, 361, 2518-2528. [CrossRef]

17. Morisaki, T.; Gross, M.; Morisaki, H.; Pongratz, D.; Zöllner, N.; Holmes, E.W. Molecular basis of AMP deaminase deficiency in skeletal muscle. Proc. Natl. Acad. Sci. USA 1992, 89, 6457-6461. [CrossRef]

18. Futema, M.; Shah, S.; Cooper, J.A.; Li, K.; Whittall, R.A.; Sharifi, M.; Goldberg, O.; Drogari, E.; Mollaki, V.; Wiegman, A.; et al. Refinement of Variant Selection for the LDL Cholesterol Genetic Risk Score in the Diagnosis of the Polygenic Form of Clinical Familial Hypercholesterolemia and Replication in Samples from 6 Countries. Clin. Chem. 2015, 61, 231-238. [CrossRef]

19. Ference, B.A.; Ginsberg, H.N.; Graham, I.; Ray, K.K.; Packard, C.J.; Bruckert, É.; Hegele, R.A.; Krauss, R.M.; Raal, F.J.; Schunkert, H.; et al. Low-density lipoproteins cause atherosclerotic cardiovascular disease. 1. Evidence from genetic, epidemiologic, and clinical studies. A consensus statement from the European Atherosclerosis Society Consensus Panel. Eur. Hear. J. 2017, 38, 2459-2472. [CrossRef]

20. Sharifi, M.; Higginson, E.; Bos, S.; Gallivan, A.; Harvey, D.; Li, K.W.; Abeysekera, A.; Haddon, A.; Ashby, H.; Shipman, K.; et al. Greater preclinical atherosclerosis in treated monogenic familial hypercholesterolemia vs. polygenic hypercholesterolemia. Atherosclerosis 2017, 263, 405-411. [CrossRef]

21. Khera, A.V.; Won, H.H.; Peloso, G.M.; Lawson, K.S.; Bartz, T.M.; Deng, X.; Morrison, A.C. Diagnostic Yield and Clinical Utility of Sequencing Familial Hypercholesterolaemia Genes in Patients with Severe Hypercholesterolaemia. J. Am. Coll. Cardiol. 2016, 67, 2578-2589. [CrossRef] [PubMed]

22. Mundal, L.; Igland, J.; Ose, L.; Holven, K.B.; Veierød, M.B.; Leren, T.P.; Retterstøl, K. Cardiovascular disease mortality in patients with genetically verified familial hypercholesterolemia in Norway during 1992-2013. Eur. J. Prev. Cardiol. 2016, 24, 137-144. [CrossRef] [PubMed]

23. Wald, D.S.; Bangash, F.A.; Bestwick, J.P. Prevalence of DNA-confirmed familial hypercholesterolaemia in young patients with myocardial infarction. Eur. J. Int. Med. 2015, 26, 127-130. [CrossRef] [PubMed]

24. De Isla, L.P.; Alonso, R.; Mata, N.; Saltijeral, A.; Muñiz, O.; Rubio-Marin, P.; Diaz-Diaz, J.L.; Fuentes, F.; De Andrés, R.; Zambón, D.; et al. Coronary Heart Disease, Peripheral Arterial Disease, and Stroke in Familial Hypercholesterolaemia. Arter. Thromb. Vasc. Boil. 2016, 36, 2004-2010. [CrossRef]

25. Paquette, M.; Dufour, R.; Baass, A. The Montreal-FH-SCORE: A new score to predict cardiovascular events in familial hypercholesterolemia. J. Clin. Lipidol. 2017, 11, 80-86. [CrossRef]

26. De Isla, L.P.; Alonso, R.; Mata, N.; Fernandez-Perez, C.; Muñiz, O.; Díaz, J.L.D.; Saltijeral, A.; Fuentes-Jiménez, F.J.; De Andrés, R.; Zambón, D.; et al. Predicting Cardiovascular Events in Familial Hypercholesterolemia. Circulation 2017, 135, 2133-2144. [CrossRef]

27. Paquette, M.; Chong, M.; Thériault, S.; Dufour, R.; Paré, G.; Baass, A. Polygenic risk score predicts prevalence of cardiovascular disease in patients with familial hypercholesterolemia. J. Clin. Lipidol. 2017, 11, 725-732. [CrossRef]

28. Besseling, J.; Huijgen, R.; Martin, S.S.; A Hutten, B.; Kastelein, J.J.; Hovingh, G.K. Clinical phenotype in relation to the distance-to-index-patient in familial hypercholesterolemia. Atherosclerosis 2016, 246, 1-6. [CrossRef]

(C) 2020 by the authors. Licensee MDPI, Basel, Switzerland. This article is an open access article distributed under the terms and conditions of the Creative Commons Attribution (CC BY) license (http://creativecommons.org/licenses/by/4.0/). 